\title{
Time-Domain Astronomy with SwIFT, FERMi and LoBSTER
}

\author{
Neil Gehrels ${ }^{1}$, Scott D. Barthelmy ${ }^{1}$, and John K. Cannizzo ${ }^{2}$ \\ ${ }^{1}$ Astroparticle Physics Laboratory, NASA Goddard Space Flight Center, \\ Greenbelt, MD 20771, USA \\ email: neil.gehrels@nasa.gov \\ ${ }^{2}$ Astroparticle Physics Laboratory, CRESST/UMBC/Goddard Space Flight Center, \\ Greenbelt, MD 20771, USA \\ Invited Talk
}

\begin{abstract}
The dynamic transient gamma-ray sky is revealing many interesting results, largely due to findings by FERMI and SwIFT. The list includes new twists on gamma-ray bursts (GRBs), a $\mathrm{GeV}$ flare from a symbiotic star, $\mathrm{GeV}$ flares from the Crab Nebula, high-energy emission from novae and supernovae, and, within the last year, a new type of object discovered by SwIFT-a jetted tidal disruption event. In this review we present highlights of these exciting discoveries. A new mission concept called LoBsTer is also described; it would monitor the X-ray sky at order-of-magnitude higher sensitivity than current missions can.
\end{abstract}

\section{Overview}

The highly variable nature of the gamma-ray sky revealed in the last few years, as well as the detection of very high energy emission from unlikely sources, have surprised the high-energy community and sent theorists scrambling back to the drawing board. Giant flares have been discovered in a source that was once thought to represent a standard measure of constant flux, and fairly benign semi-detached interacting binaries such as symbiotic stars with a WD accretor have shown $>100 \mathrm{MeV}$ emission. Such high energy emission from a WD accretor (in the galactic transient V407 Cyg, a symbiotic star) is highly unexpected given that $G M_{\mathrm{wd}} m_{\text {electron }} /\left(R_{\mathrm{wd}} k_{B}\right) \simeq 100 \mathrm{eV}$, and shows immediately that such emission cannot be associated with gravitational release of energy, either thermal or nonthermal. At the largest end of the accretor mass scale, supermassive black holes, in March 2011 SwIFT discovered Sw 1644+57 (initially GRB 110328A) which is believed to be a jetted tidal disruption event (TDE). Perhaps the greatest surprise came with the recent discovery of $\mathrm{GeV}$ flares from the Crab nebula, previously considered to be a canonical standard candle powered primarily by simple pulsar spin-down.

\section{High Energy Variability in SWIFT and FERMI}

SwIFT (Gehrels et al. 2004) carries three instruments: a wide-field Burst Alert Telescope (BAT; Barthelmy et al. 2005) which detects GRBs and positions them to arcminute accuracy, the narrow-field X-Ray Telescope (XRT; Burrows et al. 2005), and the UV-Optical Telescope (UVOT; Roming et al. 2005). BAT is a coded-aperture hard X-ray $(15-350 \mathrm{keV})$ imager with $0.5 \mathrm{~m}^{2}$ of CdZnTe detectors $(32,000$ individual sensors; $\sim 2400 \mathrm{~cm}^{2}$ effective area at $20 \mathrm{keV}$ including mask occultation) and a $1.4 \mathrm{sr}$ halfcoded field of view. XRT is a Wolter 1 grazing-incidence, imaging X-ray telescope with a $0.2-10 \mathrm{keV}$ energy range, $120 \mathrm{~cm}^{2}$ effective area at $1.5 \mathrm{keV}$, field of view of $23^{\prime} .6 \times 23^{\prime} .6$, point spread function (PSF) half-power diameter of $18^{\prime \prime}$ ( $\left.7^{\prime \prime} \mathrm{FWHM}\right)$, and sensitivity of 


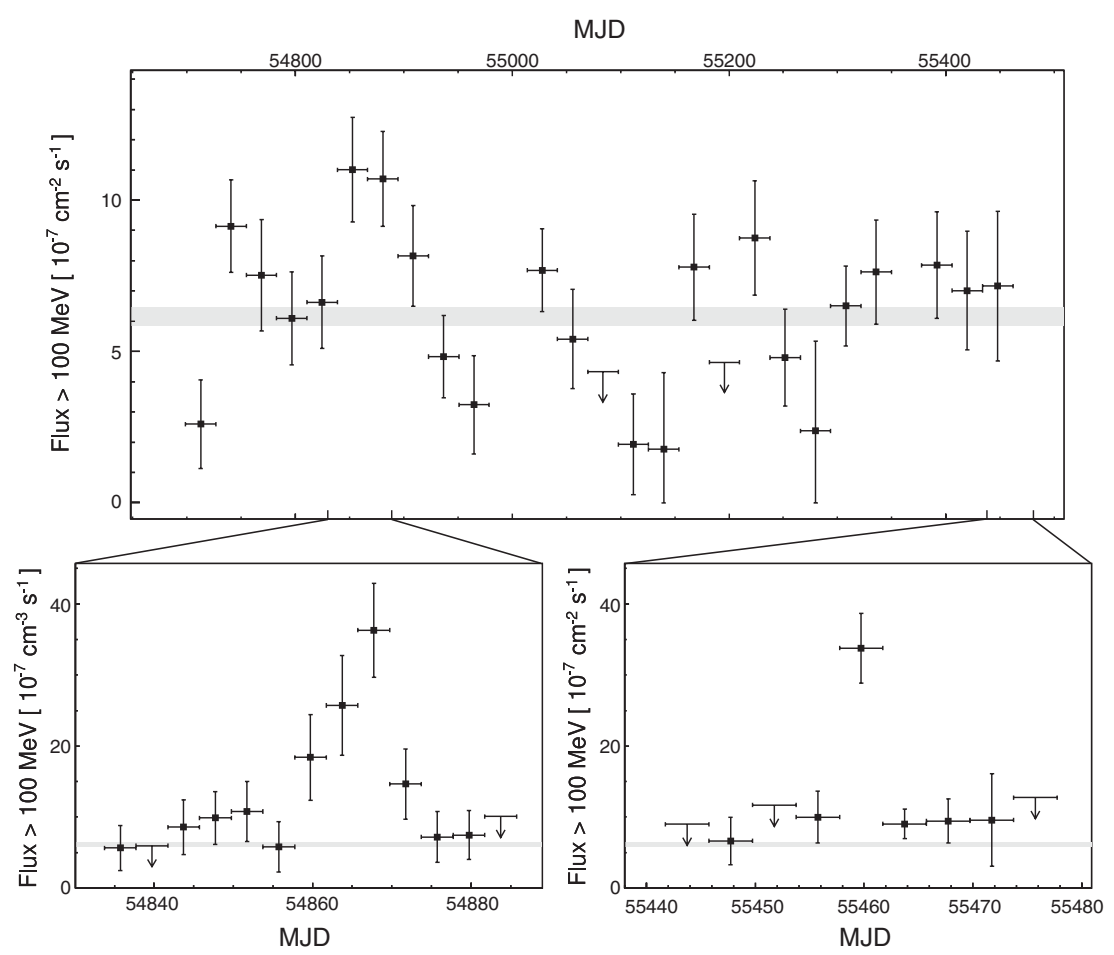

Figure 1. A light curve of the Crab nebula for photons with $E>100 \mathrm{MeV}$ as observed by FERMI/LAT (Abdo et al. 2011).

approximately $2 \times 10^{-14} \mathrm{erg} \mathrm{cm}^{-2} \mathrm{~s}^{-1}$ in $10^{4} \mathrm{~s}$. The UVOT is a modified Ritchey-Chrétien reflector with a $30-\mathrm{cm}$ aperture, $170-650 \mathrm{~nm}$ wavelength range, field of view $17^{\prime} \times 17^{\prime}$, PSF FWHM of 1.9 arcsec at $350 \mathrm{~nm}$, and sensitivity of $23^{\text {rd }}$ magnitude in white light in $10^{3} \mathrm{~s}$. The SWIFT mission was built by an international team from the US, UK, and Italy. After five years of development it was launched from Kennedy Space Center on 2004 November 20. Full normal operations commenced on 2005 April 5.

FERMI has two primary high-energy detectors: the Large Area Telescope (LAT), which operates between $20 \mathrm{MeV}$ and $\gtrsim 300 \mathrm{GeV}$ (Atwood et al. 2009) and the Gamma-Ray Burst Monitor (GBM), which operates between $8 \mathrm{keV}$ and $40 \mathrm{MeV}$ (Meegan et al. 2009). The LAT is a $3000-\mathrm{kg}$ instrument consisting of $77 \mathrm{~m}^{2}$ of silicon detectors. It is a pair conversion telescope with silicon detector tracker and caesium iodide calorimeter. The LAT scans the full sky every $3 \mathrm{hr}$; all data are made public immediately. The GBM is a scintillator-based instrument with 12 sodium iodide detectors and two bismuth germanate detectors. FERMI was launched into low-Earth orbit in 2008 June.

There are many types of objects for which variability has been observed. Galactic black-hole transients are seen to have major outbursts thought to be caused by limit cycle oscillations of the accretion disk. Those outbursts can last for hundreds of days. Novaethermonuclear explosions on the surfaces of WDs - can be quite powerful at energies of several hundred $\mathrm{MeV}$ and are thought to be providing information about the dynamics of the expanding ejecta. Flare stars are seen to vary dramatically in the $\sim 10-100 \mathrm{keV}$ bandpass, providing information about changes in the magnetic field configuration of the star. Blazars are AGN in which we view the central BH directly down the jet. There can be large flares presumably due to the introduction of accreted material near the $\mathrm{BH}$. GRBs are important sources for SWIFT and FERMI. 
Both missions continue to enlarge the data-base of known and well-categorized GRB behaviour, as well as expand our knowledge by making unexpected discoveries (Gehrels et al. 2009). Long GRBs are thought to be due the core collapse of massive, low-metallicity stars in the early Universe, whereas short GRBs are due to NS-NS mergers. Finally, one of the greatest surprises of the past several years in high-energy astrophysics has been the discovery of major, high-energy flares in the Crab nebula.

\section{Fermi/LAT and AGILE Crab Flares}

The Crab Nebula is the result of a supernova. It was observed by the Chinese in 1054 $\mathrm{AD}$; it lies at a distance $\sim 2 \mathrm{kpc}$, and has a physical extent of $\sim 3 \mathrm{pc}$. The observed power output $\dot{E}_{\text {out }}$ from the nebula, kinetic plus radiation, is $\sim 5 \times 10^{38} \mathrm{erg} \mathrm{s}^{-1}$. The expanding ejecta which make up the nebula are thought to be powered primarily by the spin-down energy from the pulsar, $\dot{E}_{\text {loss }}=I \Omega \dot{\Omega}$ (Shapiro \& Teukolsky 1983), where $I$ is the neutron star moment of inertia and $\Omega$ the rotational rate $(\sim 30 \mathrm{~Hz})$. Evaluating the loss rate assuming neutron star parameters $M=1.4 M_{\odot}, R=12 \mathrm{~km}$, and $I=1.4 \times 10^{45} \mathrm{~g}$ yields $\dot{E}_{\text {loss }}=6.4 \times 10^{38} \mathrm{erg} \mathrm{s}^{-1}$, in line with the total nebula power output. On the basis of those simple considerations, the recent finding of large GeV flares coming from the Crab Nebula was totally unexpected. Those flares (Fig. 1) have been seen with AGILE (Tavani et al. 2011) and by FERMI/LAT (Abdo et al. 2011). The synchrotron nebula increased and then decreased its power output by a factor $\sim 30$ over observations of several weeks. During those high-energy observations there was also low-energy coverage by CHANDRA, SwIFT, RXTE, and MAXI. Little or no variability was seen at other wavelengths. At the smallest temporal resolution of the observations, the $\sim 1$-hr variability time-scales imply a $\sim$ milliarcsec scale at the distance of the Crab. The luminosity of the brightest flare was $\sim 10^{39} \mathrm{erg} \mathrm{s}^{-1}$. It may be that the flares are due to magnetic reconnection in small knots within the nebula, and therefore represent localized but large perturbations in the overall energy budget. The Crab Nebula has been recently found to be variable in the low-energy gamma-ray band too (Wilson-Hodge et al. 2011). The variability lasts over time-scales of a year-much longer than the impulsive flare at high energies.

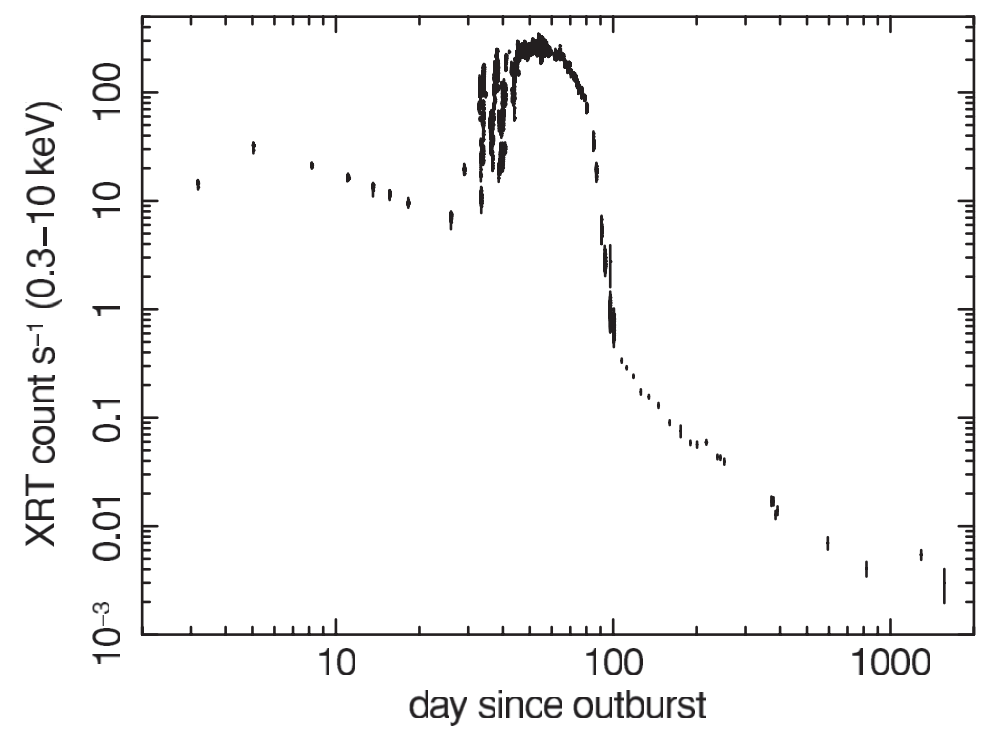

Figure 2. The 2006 nova outburst from RS Oph (Osborne et al. 2011). 


\section{Nova Studies with SwIFT}

Novae can occur in interacting binaries containing a WD accretor, and are caused by the thermonuclear detonation of accreted material on the surface of a WD (Gallagher \& Starrfield 1978). That can occur if the temperature and pressure at the base of the accumulated layer of accreted matter are in the appropriate regime. With its flexible scheduling and X-ray telescope, SwIFT has opened a new window on nova studies. To date SwIFT has observed 28 novæ. It has detected keV emission from shocked ejecta and supersoft (SS) emission from the WD surface. Extensive observations ( $\sim 400 \mathrm{ks})$ of the 2006 nova outburst from RS Oph (Fig. 2) found an unexpected SS state, and 35-s QPO (Osborne et al. 2011). Detailed analyses of SwIFT observations revealed a mass ejection of $\sim 3 \times 10^{-5} M_{\odot}$ at $\sim 4000 \mathrm{~km} \mathrm{~s}^{-1}$ into the wind of the mass-losing red giant companion in the system.

\section{Swift J1644+57 - The First Jetted TDE}

Sw 1644+57 was triggered as GRB 110328A (Burrows et al. 2011). Evidence connecting the X-ray/gamma-ray source with a galactic nucleus (Berger et al. 2011) was confirmed by precise HST and CHANDRA localizations (Fruchter et al. 2011; Levan et al. 2011). The host dwarf (non-AGN type) galaxy lies at $z=0.35$ (Levan et al. 2011). The strong circumstantial evidence associating the X-ray emission with the centre of the dwarf galaxy suggests a TDE (Bloom et al. 2011). Long term monitoring showed its X-ray light curve to be quite different from a normal GRB (Shao et al. 2011). Fig. 3 reproduces the longterm light curve. The bottom panel shows the flux binned in time as 0.125 dex. The two superposed decay segments indicate the putative theoretical decay laws for stellar debris accretion and a long-term accretion disk (Cannizzo et al. 2011). It is surprising that such events have not been identified earlier, but now that we have some experience to guide us, a second such event has already been tentatively identified (Cenko et al. 2011).

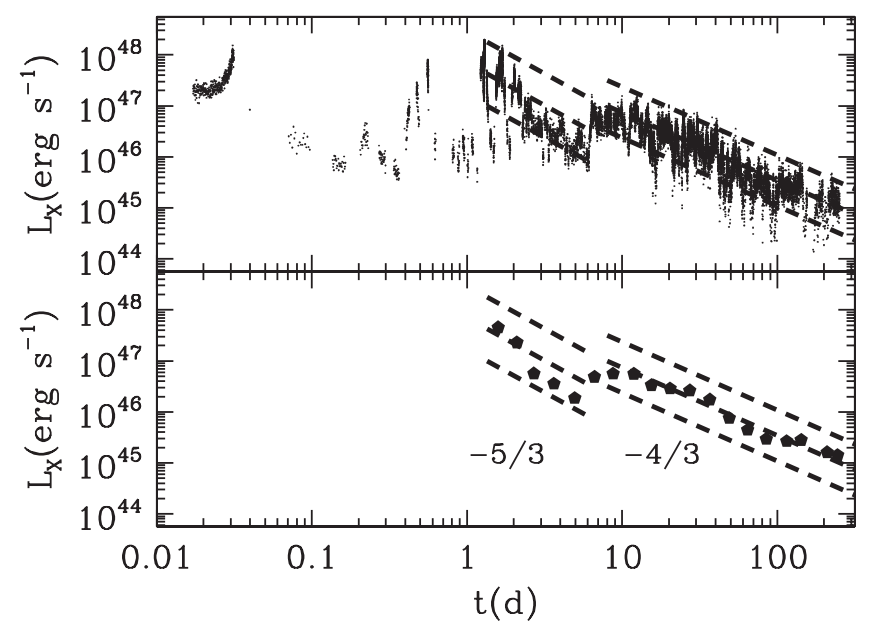

Figure 3. SwIFT/XRT light curve of $\mathrm{Sw} 1644+57$. The $-5 / 3$ decay law is indicative of the direct accretion of stellar debris, whereas the $-4 / 3$ would be characteristic of a long term accretion disk (after Cannizzo et al. 2011). 


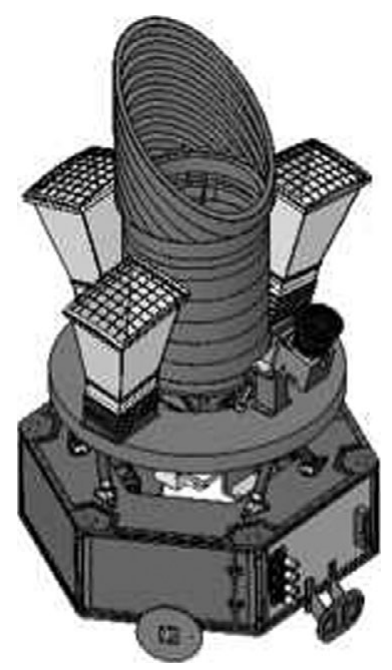

Figure 4. LOBSTER observatory.

\section{Lobster Mission Concept}

The tantalizing glimpses afforded us to date by serendipitous sources such as Sw $1644+57$ have underscored the need for more efficient discovery techniques in order to increase the future rate of discovery of such objects. To that end, LoBsTER (Fig. 4) is a mission concept led by Goddard Space Flight Center, MIT, the University of Leicester and the University of Arizona to combine an X-ray wide-field imager (WFI) with a narrow-field IR telescope (IRT). The underlying strategy is similar to that of SwIFT: a detection at high energies, followed up by more detailed observations. The WFI has a combined 0.5 -sr field of view that covers $\sim 50 \%$ of the sky every 3 hours in multiple pointings. It is based on CCD technology and lobster-eye microchannel optics (Angel 1979). The IRT has a 40 -cm diameter mirror, a wavelength range of $0.6-2.1 \mu$, and is capable of multiband photometry and $\lambda / \delta \lambda=30$ slit spectroscopy. An estimation of the rate for jetted TDEs in an instrument like LoBsTER, based on Sw $1644+57$, is $\sim 1-2 \mathrm{yr}^{-1}$

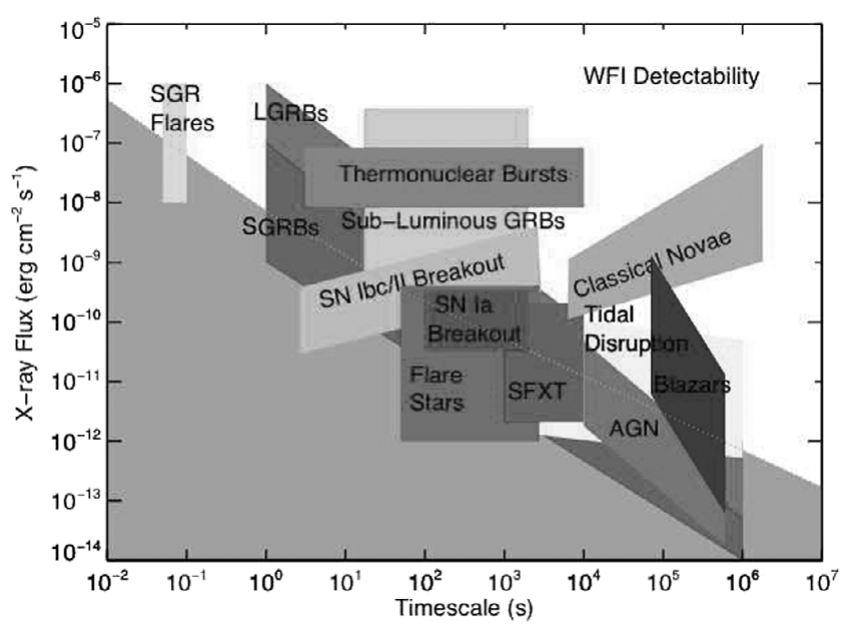

Figure 5. LOBSTER/WFI transient detectability. 
(Cannizzo et al. 2011). Fig. 5 indicates in more generality the parameter space for LoBSTER transients.

LOBSTER will also provide capabilities for a major step forward in studies of the highredshift universe using GRBs. The prompt emission is detected by the WFIs and observed in X-ray afterglow by the WFIs, and in infrared afterglow by the IRT. Improvements over SwIFT include: (1) a more sensitive wide-field instrument, (2) an infrared follow-up telescope and (3) slit spectroscopy by the follow-up telescope. The mission is expected to detect more than 25 bursts per year at redshift $z>5$, and to extend detections to $z>12$.

\section{Summary}

The high-energy sky is dominated by transient sources, which is somewhat surprising given our prior expectations. This is especially true with the unexpected discovery of large flares in the Crab Nebula, which has been considered historically as a fiducial measuring stick, giving rise to its own unit of flux, "the Crab". FERMI and SwIFT continue to monitor the sky, continuously and with sensitivity. Those missions have yielded important results: in the SWIFT era it has been definitively established, through localizations based on the prompt SwIFT/BAT discoveries, that long and short GRBs have different origins. That result was reached by identifying differences in their progenitor galaxies, and through studying offsets within those hosts. In 2011 March a new type of object was discovered: a tidal disruption event viewed down the jet. Nova and supernova observations are also providing important clues about their high-energy transient behaviour, thereby helping to disclose their true nature.

\section{References}

Abdo, A. A., et al. 2011, Science, 331, 739

Angel, J. R. P. 1979, ApJ, 233, 364

Atwood, W. B., et al. 2009, ApJ, 697, 1071

Barthelmy, S. D., et al. 2005, Space Sci. Rev., 120, 143

Berger, E., et al. 2011, GCN Circ., 11854, 1

Bloom, J. S., et al. 2011, Science, 333, 203

Burrows, D. N., et al. 2005, Space Sci. Rev., 120, 165

Burrows, D. N., et al. 2011, Nature, 476, 421

Cannizzo, J. K., Troja, E., \& Lodato, G. 2011, ApJ, 742, 32

Cenko, S. B., et al. 2011, arXiv, 1107.5307v1

Fruchter, A. F., et al. 2011, GCN Circ., 11881, 1

Gallagher, J. S. \& Starrfield, S. 1978, ARAA, 16, 171

Gehrels, N., et al. 2004, ApJ, 611, 1005

Gehrels, N., Ramirez-Ruiz, E., \& Fox, D. B. 2009, ARAA, 47, 567

Levan, A. J., et al. 2011, Science, 333, 199

Meegan, C., et al. 2009, ApJ, 702, 791

Osborne, J. P., et al. 2011, ApJ, 727, 124

Roming, P. W. A., et al. 2005, Space Sci. Rev., 120, 95

Shao, L., Zhang, F.-W., Fan, Y.-Z., \& Wei, D.-M. 2011, ApJ, 734, 33

Shapiro, S. L. \& Teukolsky, S. A. 1983, Black Holes, White Dwarfs, and Neutron Stars: The Physics of Compact Objects (New York: John Wiley \& Sons)

Tavani, M., Bulgarelli, A., \& Vittorini, V., et al. 2011, Science, 331, 736

Wilson-Hodge, C. A., et al. 2011, ApJ, 727, 40 